\title{
Stability of Accretion Shocks with a Composite Cooling Function
}

\author{
Curtis J. Saxton ${ }^{1}$, Kinwah $\mathrm{Wu}^{1}$ and Helen Pongracic ${ }^{2}$ \\ ${ }^{1}$ Special Research Centre for Theoretical Astrophysics, School of Physics, \\ University of Sydney, NSW 2006, Australia \\ 2 Air Operations Division, Aeronautical and Maritime \\ Research Laboratory, DSTO, Vic. 3001, Australia \\ saxton@physics.usyd.edu.au
}

Received 1996 December 19, accepted 1997 April 4

\begin{abstract}
Stability of accretion onto magnetic white dwarfs is investigated via perturbation analysis. We consider a cooling function with composite power laws, and the power-law indices are appropriate for bremsstrahlung and optically thick cyclotron cooling. We find that the presence of cyclotron cooling generally suppresses the instability, consistent with numerical simulations by other investigators.
\end{abstract}

Keywords: accretion — shock waves - stars: close binaries - stars: white dwarfs

\section{Introduction}

Theoretical studies (e.g. Langer, Chanmugam \& Shaviv 1981; Chevalier \& Imamura 1982; Imamura 1985) have shown that accretion onto white dwarfs is thermally unstable if bremsstrahlung radiation is the cooling process. The accretion shock, which is formed near the white dwarf surface when supersonic accretion matter becomes subsonic, is thereby driven to oscillate, with a typical oscillation timescale similar to the cooling timescale of the shock-heated matter. These studies stimulated searches for fast photometric variabilities in accreting white dwarfs and led to the subsequent discovery of optical quasi-periodic oscillations (QPOs) in the AM Herculis systems V834 Cen, AN UMa, EF Eri and VV Pup (Middleditch 1982; Mason et al. 1983; Larsson 1985, 1987). (AM Herculis systems are binaries consisting of a Rochelobe-filling red dwarf and a magnetic white dwarf, both in synchronous rotation with the orbit - see the review by Cropper 1990.) While these four systems were found to show QPOs, null detections were reported for other AM Herculis systems.

The typical shock temperature of accreting white dwarfs is $\sim 10^{8} \mathrm{~K}$, and the white dwarf magnetic fields in $\mathrm{AM}$ Herculis systems are $B \approx 10-70 \mathrm{MG}$. Cyclotron cooling is therefore also an important process in the post-shock emission regions. For sufficiently low accretion rates, cyclotron cooling may dominate bremsstrahlung cooling (Lamb \& Masters 1979; King \& Lasota 1979) and hence alter the stability properties of the accretion shock. The effects of cyclotron cooling were investigated by Langer, Chanmugam \& Shaviv (1982) and Chanmugam, Langer \& Shaviv (1985). Their numerical simulations show damping of the shock oscillations when cyclotron cooling is present. Moreover, the oscillation frequency increases with the magnetic field strength (Wu, Chanmugam \& Shaviv 1992).

Although the numerical studies have been successful in describing the instabilities in the accretion flow, they fail to explain why the oscillations are excited and why certain modes can persist while others cannot. There are also discrepancies in the results obtained by calculations using different numerical formulations (cf. Langer, Chanmugam \& Shaviv 1982; Imamura 1985).

Here we investigate the stability properties of accreting shocks with cyclotron cooling by considering an analytic approach. Perturbation analyses of accretion shocks have been carried by many authors (e.g. Chevalier \& Imamura 1982; Bertschinger 1986; Houck \& Chevalier 1992; Toth \& Draine 1993; Dgani \& Soker 1994). In these studies, the cooling function is generally taken to be a single power-law form. Although it is adequate for the situation where bremsstrahlung cooling is the only process, it is insufficient for the study of the competing effects of two cooling processes with different stability properties. Here, we consider a formulation generalised from that given in Chevalier \& Imamura (1982), such that the total cooling is represented by a composite function consisting of two power-law terms. The first term is the bremsstrahlung cooling function, which has the form $\rho^{2} T^{\frac{1}{2}}$, where $\rho$ and $T$ are density and temperature respectively. The second term is the effective cyclotron cooling function, mimicked by a function of the form $\rho^{a} T^{b}$, where $a$ and $b$ are power-law indices to be determined (Appendix A; see also Wu, Chanmugam \& Shaviv 1994). With this composite function, we obtain the hydrodynamic equations and carry out a perturbation analysis to determine the oscillation modes and their stability properties. 


\section{Accretion onto Magnetic White Dwarfs}

The height of accretion shocks (i.e. the thickness of post-shock regions), $x_{s}$, of magnetic white dwarfs are generally $\ll R_{w d}$, where $R_{w d}$ is the white dwarf radius. For typical accretion rates $\left(\sim 10^{16} \mathrm{~g} \mathrm{~s}^{-1}\right)$, the ram pressure of the accretion matter near the white dwarf surface is less than the magnetic stress. As the accretion flow is channelled by the field lines, it can be approximated by a one-dimensional planar model. The time-dependent continuity and momentum equations are therefore

$$
\frac{\partial \rho}{\partial t}+\rho \frac{\partial v}{\partial x}+v \frac{\partial \rho}{\partial x}=0
$$

and

$$
\rho\left(\frac{\partial v}{\partial t}+v \frac{\partial v}{\partial x}\right)+\frac{\partial P}{\partial x}=0
$$

where $P$ is the pressure and $v$ is the velocity. If we assume that the temperature of the accretion matter is proportional to $P / \rho$ and the matter has an adiabatic index of $5 / 3$, the energy equation is then

$$
\begin{aligned}
& \frac{\partial P}{\partial t}+v \frac{\partial P}{\partial x}-\frac{5}{3} \frac{P}{\rho}\left(\frac{\partial \rho}{\partial t}+v \frac{\partial \rho}{\partial x}\right) \\
= & -\frac{2}{3} \mathcal{C} \rho^{2}\left(\frac{P}{\rho}\right)^{\frac{1}{2}}\left[1+\epsilon_{s}\left(\frac{P}{P_{s}}\right)^{\alpha}\left(\frac{\rho}{\rho_{s}}\right)^{-\beta}\right]
\end{aligned}
$$

(cf. Wu 1994), where $\epsilon_{s}$ is the effective ratio of the bremsstrahlung cooling timescale to the cyclotron cooling timescale at the shock surface. In the above equation and hereafter, the subscript ' $s$ ' denotes parameters and variables at the shock surface. The coefficient

$$
\mathcal{C}=\left(2 \pi k_{B} / 3 m_{e}\right)^{\frac{1}{2}}\left(2^{5} \pi e^{6} / 3 h m_{e} c^{3}\right)\left(\mu / k_{B} m_{p}^{3}\right)^{\frac{1}{2}} g_{B}
$$

corresponds to bremsstrahlung cooling, where $k_{B}$ is the Boltzmann constant, $h$ the Planck constant, $c$ the velocity of light, $e$ the electron charge, $m_{e}$ the electron mass, $m_{p}$ the proton mass, $\mu$ the mean molecular weight of the gas and $g_{B}$ the Gaunt factor (see Rybicki \& Lightman 1979). The numerical value of $\mathcal{C}$ is $3.9 \times 10^{16}$ in c.g.s. units, assuming that $\mu=0.5$ and $g_{B}=1$. For typical parameters of AM Herculis systems, $\alpha \approx 2$ and $\beta \approx 3.85$ (Appendix A).

We assume a 'stationary wall' boundary condition at the white dwarf surface, so that the lower boundary condition is $v=0$. At the shock surface, we assume the strong shock condition, so that $v=-v_{f f} / 4, \rho=4 \rho_{a}$ and $P=3 \rho_{a} v_{f f}^{2}$, where $v_{f f}$ is the free-fall velocity at the white dwarf surface and $\rho_{a}$ is density above the shock. With the boundary conditions specified, a closed-form steady- state solution $\left\{P_{0}, \rho_{0}, v_{0}\right\}$ to equations (1), (2) and (3) and a steady-state shock height $x_{s 0}$ can be obtained (Wu, Chanmugam \& Shaviv 1994; see also Aizu 1973 and Chevalier \& Imamura 1982 for the cases of single power-law cooling functions).

\section{Perturbation Analysis}

Following Chevalier \& Imamura (1982), we consider a perturbation

$$
\frac{\mathrm{d} x_{s}}{\mathrm{~d} t}=v_{s 1} \exp (\omega t)
$$

where $v_{s 1}$ is the perturbed velocity of the shock surface, taken to be real, and $\omega$ is the complex eigenvalue which determines the stability. (Hereafter, the subscripts ' 0 ' and ' 1 ' denote the steady state and the perturbed quantities respectively.) The time-dependent shock height is

$$
x_{s}=x_{s 0}+x_{s 1} \exp (\omega t),
$$

where $\omega=v_{s 1} / x_{s 1}$. We also assume that the perturbed variables are given by

$$
\begin{gathered}
P(\xi, t)=P_{0}(\xi)+P_{1}(\xi) \exp (\omega t), \\
\rho(\xi, t)=\rho_{0}(\xi)+\rho_{1}(\xi) \exp (\omega t), \\
v(\xi, t)=v_{0}(\xi)+v_{1}(\xi) \exp (\omega t),
\end{gathered}
$$

where $\xi=x / x_{s}$. Substituting equations (6), (7) and (8) into equations (1), (2) and (3) and considering the dimensionless variables $\tau=-v_{0} / v_{f f}$, $\zeta=x_{s 0} \omega \rho_{1} / v_{s 1} \rho_{a}, \pi=P_{1} / \rho_{a} v_{f f} v_{s 1}, \eta=v_{1} / v_{s 1}$ and $\delta=x_{s 0} \omega / v_{f f}$, we obtain three complex, linear perturbed equations

$$
\begin{aligned}
& -\zeta \frac{\mathrm{d} \xi}{\mathrm{d} \tau}+\frac{\tau}{\delta} \frac{\mathrm{d} \zeta}{\mathrm{d} \tau}-\frac{1}{\tau} \frac{\mathrm{d} \eta}{\mathrm{d} \tau}-\frac{\xi}{\tau^{2}}+\frac{\zeta}{\delta}+\frac{\eta}{\tau^{2}}=0 \\
& -\delta \eta \frac{\mathrm{d} \xi}{\mathrm{d} \tau}+\tau \frac{\mathrm{d} \eta}{\mathrm{d} \tau}-\tau \frac{\mathrm{d} \pi}{\mathrm{d} \tau}-\xi+\eta-\frac{\zeta \tau^{2}}{\delta}=0 \\
& -\pi \delta \frac{\mathrm{d} \xi}{\mathrm{d} \tau}-\frac{5}{3}(1-\tau) \frac{\mathrm{d} \eta}{\mathrm{d} \tau}+\tau \frac{\mathrm{d} \pi}{\mathrm{d} \tau} \\
& -\xi+\eta+\frac{5}{3} \pi-\frac{(5-8 \tau)}{3} \frac{1}{\delta} \\
& =\frac{1}{3}(5-8 \tau)\left\{\frac{1}{2(1-\tau)}\left[\frac{1+(1+2 \alpha) \epsilon_{s} f(\tau)}{1+\epsilon_{s} f(\tau)}\right]\right. \\
& \left.+\frac{3}{2}\left[\frac{1+\left(1-\frac{2}{3} \beta\right) \epsilon_{s} f(\tau)}{1+\epsilon_{s} f(\tau)}\right] \frac{\tau \zeta}{\delta}\right\}
\end{aligned}
$$

where

$$
f(\tau)=\frac{4^{\alpha}+\beta}{3^{\alpha}}(1-\tau)^{\alpha} \tau^{\beta}
$$


Table 1. The eigenvalues for the cases $\epsilon_{\mathrm{s}}=0,1$ and 10

\begin{tabular}{|c|c|c|c|c|c|c|}
\hline \multirow[b]{2}{*}{$n$} & \multicolumn{2}{|c|}{$\epsilon_{s}=0$} & \multicolumn{2}{|c|}{$\epsilon_{s}=1$} & \multicolumn{2}{|c|}{$\epsilon_{s}=10$} \\
\hline & $\delta_{R}$ & $\delta_{I}$ & $\delta_{R}$ & $\delta_{I}$ & $\delta_{R}$ & $\delta_{I}$ \\
\hline 1 & $-0 \cdot 010$ & $0 \cdot 305$ & -0.061 & 0.322 & $-0 \cdot 156$ & 0.309 \\
\hline 2 & $0 \cdot 047$ & 0.887 & -0.036 & 0.838 & -0.077 & 0.707 \\
\hline 3 & 0.061 & $1 \cdot 504$ & 0.003 & $1 \cdot 419$ & -0.119 & $1 \cdot 161$ \\
\hline 4 & 0.085 & $2 \cdot 107$ & 0.033 & 1.976 & -0.065 & $1 \cdot 637$ \\
\hline 5 & 0.088 & $2 \cdot 723$ & $0 \cdot 028$ & $2 \cdot 553$ & -0.077 & $2 \cdot 057$ \\
\hline 6 & $0 \cdot 107$ & $3 \cdot 331$ & 0.051 & $3 \cdot 124$ & -0.073 & $2 \cdot 553$ \\
\hline 7 & $0 \cdot 105$ & $3 \cdot 944$ & 0.046 & $3 \cdot 691$ & -0.045 & $2 \cdot 986$ \\
\hline 8 & 0.121 & $4 \cdot 555$ & 0.063 & $4 \cdot 270$ & -0.069 & $3 \cdot 456$ \\
\hline
\end{tabular}

Equations (9), (10) and (11) can be separated into six real decoupled linearised equations. With the boundary conditions $v_{1}=0$ at the white dwarf surface and $v_{1}=3 v_{s 1} / 4, \rho_{1}=0$ and $P_{1}=3 \rho_{a} v_{f f} v_{s 1} / 2$ at the shock surface (see Appendix B), the eigenvalues $\delta$, and hence $\omega$, can readily be obtained.

\section{Results and Discussions}

The differential equations are solved using the Runge-Kutta method, with integration from the shock surface to the white dwarf surface. The eigenvalues $\delta$ are obtained when the solution at the white dwarf surface matches the 'stationary wall' boundary condition (i.e. $v_{1}=0$ ).

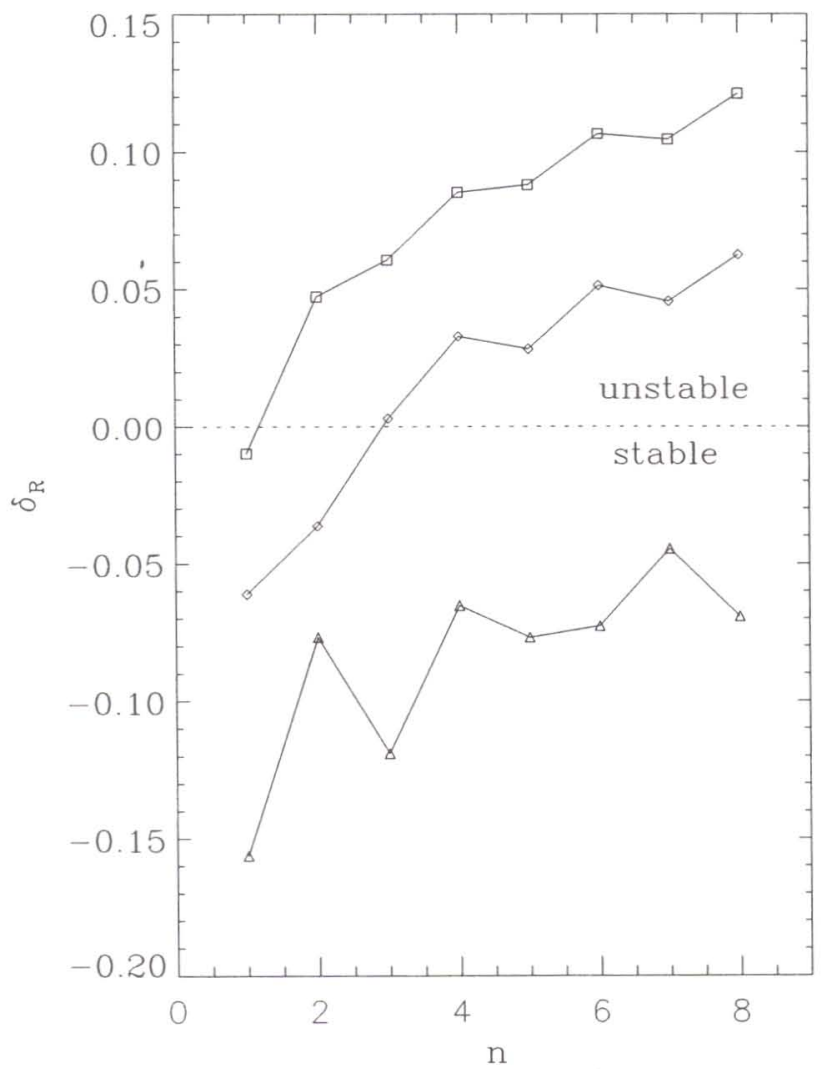

Figure 1-Real part of the eigenvalue, $\delta_{R}$, as a function of the harmonic number $n$. The squares, diamonds and triangles correspond to the cases $\epsilon_{s}=0,1$ and 10 respectively.

In Table 1 , the real and imaginary parts of the eigenvalues $\left(\delta_{R}\right.$ and $\delta_{I}$ respectively) of the first eight harmonics for the cases $\epsilon_{s}=0,1$ and 10 are shown. For $\epsilon_{s}=0$, only bremsstrahlung cooling is present; for $\epsilon_{s}=1$, cyclotron cooling and bremsstrahlung cooling have the same efficiency at the shock surface; and for $\epsilon_{s}=10$, cyclotron cooling is ten times more efficient than bremsstrahlung cooling at the shock surface, i.e. the accretion shock can be considered as being dominated by cyclotron cooling.

In Figure $1, \delta_{R}$ is plotted against $n$, the harmonic number. When bremsstrahlung is the only cooling process $\left(\epsilon_{s}=0\right)$, the first harmonic $(n=1)$ is linearly stable $\left(\delta_{R}<0\right)$, and the higher harmonics are unstable $\left(\delta_{R}>0\right)$ (see also Chevalier \& Imamura 1982).

For $\epsilon_{s}=1$, the first harmonic is stable as for the case $\epsilon_{s}=0$, but the second harmonic is also stable. The higher harmonics are, however, unstable, and the corresponding values for $\delta_{R}$ are smaller than those for the previous case. For a sufficiently high magnetic field, such as $\epsilon_{s}=10$, the first eight harmonics we have obtained are stable. Thus, our perturbation analyses show that the presence of cyclotron cooling tends to stabilise the shock oscillations, which is consistent with the results from the numerical studies of Chanmugam, Langer \& Shaviv (1985).

In Figure 2, we plot $\delta_{I}$ against $n$. Clearly there is a linear relationship whereby $\delta_{I}$ increases with $n$ for all three cases, and $\delta_{I}$ is approximately given by

$$
\delta_{I}=\left(n-\frac{1}{2}\right) \delta_{I O}+\delta_{C},
$$

where $\delta_{I O}=0.6087, \quad 0.5666$ and 0.4526 and $\delta_{C}=-0.0153,0.0077$ and 0.0477 for $\epsilon_{s}=0$, 1 and 10 respectively. This is similar to a pipe which is open at one end, for which the frequencies of the harmonics are given by $(n-0.5) f_{o} / 2+f_{c}$, where $f_{o}$ and $f_{c}$ are constants.

It is worth noting that the smaller the value of $\epsilon_{s}$, the steeper the slope of the $\delta_{I}$ vs $n$ graph. If we define the cooling time as $t_{c o o l}=x_{s o} / v_{f f}$, then from the definition of $\delta$ we have $\omega_{I}$ (the imaginary part of $\omega$ ) proportional to $t_{\text {cool }}^{\theta}$, where $\theta>1$. Thus, our calculations show that the imaginary part of the eigenvalue $\left(\delta_{I}\right)$ is determined by both the cooling function and the boundary conditions. Although the 
real part $\left(\delta_{R}\right)$ obviously depends on the details of the cooling function, whether the boundary condition is crucial still needs to be investigated.

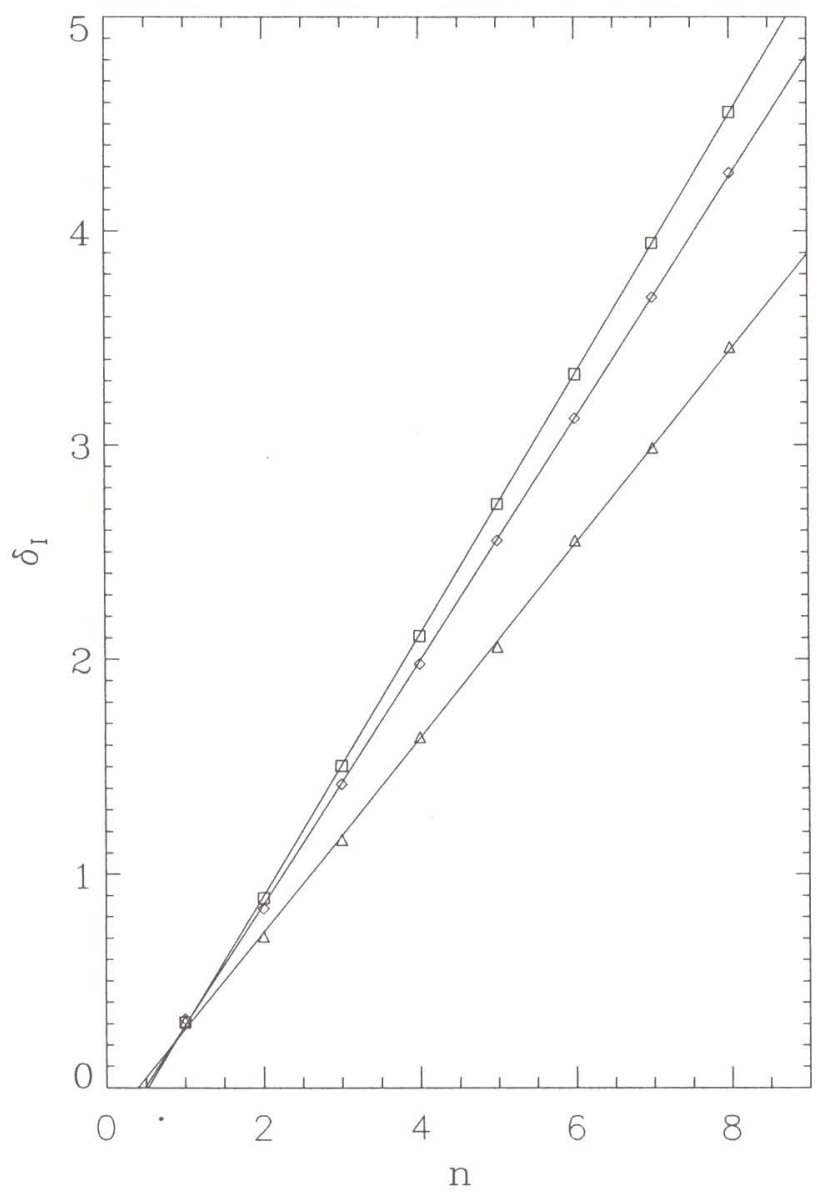

Figure 2-Same as Figure 1 but for $\delta_{I}$.

Using the expression for $\epsilon_{s}$ given in Appendix A, one can estimate that for typical accretion parameters of $\mathrm{AM}$ Herculis systems, $B \approx 50 \mathrm{MG}$ when $\epsilon_{s}=10$. The result that the accretion shock is stable for $\epsilon_{s} \gtrsim 10$ implies QPOs of $\sim 1 \mathrm{~Hz}$ are suppressed when $B \gtrsim 50 \mathrm{MG}$. For AM Herculis systems with a weaker magnetic field ( $\lesssim 30 \mathrm{MG}$ ), the higher oscillation modes are unstable, despite the lower oscillation modes being stable. However, the high oscillation modes have high frequencies and are difficult to excite. The null detection of $1 \mathrm{~Hz}$ QPOs in some AM Herculis systems may be due to insufficient instrumental sensitivity to detect the high-frequency modes.

\section{Conclusions}

We have considered a perturbation analysis of accretion shocks with a composite cooling function, in which there is a bremsstrahlung cooling term and a power-law term mimicking the effects of optically thick cyclotron cooling. We have found that in the limit of zero cyclotron cooling the first harmonic of the shock oscillation is stable while the higher harmonics are unstable. For the case with moderately strong cyclotron cooling $\left(\epsilon_{s}=1\right)$, the first two harmonics are stable but the higher harmonics are unstable. For very strong cyclotron cooling $\left(\epsilon_{s}=10\right)$, the first eight harmonics we have obtained are stable. The presence of cyclotron cooling therefore stabilises the shock oscillations.

\section{Acknowledgment}

We thank L. Ball, S. Johnston, E. Rowe and M. Wardle for discussions and comments on the manuscript. This work is partially supported by an ARC Australian Research Fellowship awarded to KW.

Aizu, K. 1973, Prog. Theor. Phys., 49, 1184

Bertschinger, E. 1986, ApJ, 304, 154

Chanmugam, G., Langer, S. H., \& Shaviv, G. 1985, ApJ, 299, L87

Chevalier, R. A., \& Imamura, J. N. 1982, ApJ, 261, 543

Cropper, M. 1990, Space Sci. Rev., 54, 195

Dgani, R., \& Soker, N. 1994, ApJ, 434, 262

Houck, J. C., \& Chevalier, R. A. 1992, ApJ, 395, 592

Imamura, J. N. 1985, ApJ, 296, 128

King, A. R., \& Lasota, J. P. 1979, MNRAS, 188, 653

Lamb, D. Q., \& Masters, A. R. 1979, ApJ, 234, L117

Langer, S. H., Chanmugam, G., \& Shaviv, G. 1981, ApJ, $245, \mathrm{~L} 23$

Langer, S. H., Chanmugam, G., \& Shaviv, G. 1982, ApJ, 258,289

Larsson, S. 1985, A\&A, 145, L1

Larsson, S. 1987, A\&A, 181, L15

Mason, K. O., et al. 1983, ApJ, 264, 575

Middleditch, J. 1982, ApJ, 257, L71

Rybicki, G. B., \& Lightman, A. P. 1979, Radiative Processes in Astrophysics (New York: Wiley)

Toth, G., \& Draine, B. T. 1993, ApJ, 413, 176

Wada, T., et al. 1980, Prog. Theor. Phys., 64, 1986

Wu, K. 1994, PASA, 11, 61

Wu, K., Chanmugam, G., \& Shaviv, G. 1992, ApJ, 397, 232

Wu, K., Chanmugam, G., \& Shaviv, G. 1994, ApJ, 426, 664

\section{Appendix A. The Composite Cooling Function}

We assume that the local total cooling function in the post-shock region is given by

$$
\begin{aligned}
\Lambda_{t o t} & =\Lambda_{b r}+\Lambda_{c y c} \\
& \approx \Lambda_{b r}\left(1+\frac{t_{b r}}{t_{c y c}}\right) \\
& =\Lambda_{b r}\left[1+\epsilon_{s}\left(\frac{P}{P_{s}}\right)^{\alpha}\left(\frac{\rho_{s}}{\rho}\right)^{\beta}\right],
\end{aligned}
$$

where $\Lambda_{b r}$ is the bremsstrahlung cooling function, $\Lambda_{c y c}$ the effective cyclotron cooling function, $t_{b r}$ the bremsstrahlung cooling timescale, $t_{c y c}$ the cyclotron cooling timescale, the subscript ' $s$ ' denotes the values at the shock surface, and $\epsilon_{s}, \alpha$ and $\beta$ are constants to 
be determined. The bremsstrahlung cooling function is $\Lambda_{b r}=1.4 \times 10^{-27} n_{e} n_{p} T^{\frac{1}{2}}$ erg $\mathrm{cm}^{-3} \mathrm{~s}^{-1}$ (e.g. Rybicki \& Lightman 1979), and the bremsstrahlung cooling timescale is

$$
t_{b r}=\frac{3}{2} \frac{\left(n_{e}+n_{p}\right) k T}{\Lambda_{b r}},
$$

where $n_{e}$ and $n_{p}$ are the electron and proton number density respectively.

We assume that the optically thick cyclotron radiation has a Rayleigh-Jeans spectrum up to a critical frequency $\omega^{*}$, and photons with frequencies beyond $\omega^{*}$ make an insignificant contribution to the cooling process and can be neglected. The angle-averaged cyclotron luminosity is therefore

$$
L_{c y c} \approx \frac{A^{2} k T\left[\omega^{*}(x)\right]^{3}}{12 \pi^{2} c^{2}}
$$

(Langer, Chanmugam \& Shaviv 1982), where $c$ is the speed of light, and $A$ and $x$ are the effective area and the thickness of the emission region respectively. For parameters appropriate for accretion shocks in magnetic cataclysmic variables

$$
\omega^{*}(x) \approx 9.87 \omega_{c}\left(\frac{\Lambda}{10^{7}}\right)^{0.05}\left(\frac{T}{10^{8} \mathrm{~K}}\right)^{0.5},
$$

(Wada et al. 1980), where $\Lambda=4 \pi e n_{e} x / B$ is the dimensionless plasma size parameter, $\omega_{c}=e B / m_{e} c$ the cyclotron frequency, $m_{e}$ the electron mass, and $e$ the electron charge. The effective cyclotron cooling timescale is therefore

$$
t_{c y c} \approx \frac{3}{2}\left(n_{e}+n_{p}\right) k T \frac{d x}{d L_{c y c}}
$$

(see Langer, Chanmugam \& Shaviv 1982). As $L_{c y c} \propto\left[\omega^{*}(x)\right]^{3} T$,

$$
\frac{d}{d x} L_{c y c} \propto\left[\omega^{*}(x)\right]^{3} T \propto n_{e}^{0 \cdot 15} T^{2 \cdot 5} .
$$

If we assume that $n_{e}=n_{p}$, then $t_{b r} \propto T^{0 \cdot 5} n_{e}^{-1}$ and $t_{c y c} \propto T^{-1 \cdot 5} n_{e}^{0 \cdot 85}$. It follows that

$$
\frac{t_{b r}}{t_{c y c}} \propto \frac{T^{2}}{n_{e}^{1 \cdot 85}} .
$$

Since $P / \rho \propto T$ and $\rho \propto n_{e}$, we have

$$
\frac{t_{b r}}{t_{c y c}}=\frac{t_{b r, s}}{t_{c y c, s}}\left(\frac{P}{P_{s}}\right)^{2 \cdot 0}\left(\frac{\rho_{s}}{\rho}\right)^{3 \cdot 85}
$$

Thus, $\alpha=2 \cdot 0, \beta=3 \cdot 85$ and $\epsilon_{s}=t_{b r, s} / t_{c y c, s}$, the ratio of the bremsstrahlung cooling timescale to the cyclotron cooling timescale at the shock surface. In terms of the parameters at the shock surface, the ratio is

$$
\begin{aligned}
\epsilon_{s} \approx 9 & \times 10^{-3}\left(\frac{B}{10 \mathrm{MG}}\right)^{2 \cdot 85}\left(\frac{T_{s}}{10^{8} \mathrm{~K}}\right)^{2} \\
& \times\left(\frac{n_{s}}{10^{16} \mathrm{~cm}^{-3}}\right)^{-1 \cdot 85}\left(\frac{x_{s}}{10^{7} \mathrm{~cm}}\right)^{-0 \cdot 85}
\end{aligned}
$$

(Wu, Chanmugam \& Shaviv 1994), where $B$ is the magnetic field, $T_{s}$ the shock temperature, $n_{s}$ the electron number density at the shock surface, and $x_{s}$ the shock height.

\section{Appendix B. The Perturbed Boundary Conditions}

Consider a reference frame which is co-moving with the shock surface. Let the subscripts ' 1 ' and ' 2 ' denote the quantities in the pre-shock and the post-shock regions respectively, and the 'prime' and 'un-prime' denote the observer's and the new reference frame (co-moving with the shock surface) respectively. If the velocity in the observer's frame is $u$, then the velocity in the new reference frame is

$$
u^{\prime}=u-\frac{d x_{s}}{\mathrm{~d} t} .
$$

From equation (4), we therefore obtain the velocity of the accretion matter,

$$
u_{1}^{\prime}=u_{1}-v_{s 1} e^{\omega t} .
$$

From the continuity equation we have

$$
\rho_{1}{ }^{\prime} u_{1}^{\prime}=\rho_{2}{ }^{\prime} u_{2}{ }^{\prime} .
$$

Since $\rho_{1}{ }^{\prime}=\rho_{1}=\rho_{a}$ (where $\rho_{a}$ is the density above the shock surface), we have $u_{2}{ }^{\prime}=\rho_{a} u_{1}{ }^{\prime} / \rho_{2}{ }^{\prime}$. For a strong shock, $\rho_{2}{ }^{\prime}=4 \rho_{1}{ }^{\prime}=4 \rho_{a}$. It follows that

$$
\begin{aligned}
& u_{2}{ }^{\prime}=\frac{1}{4} u_{1}{ }^{\prime}=\frac{1}{4}\left(u_{1}-v_{s 1} e^{\omega t}\right) \\
& u_{2}=u_{2}{ }^{\prime}+v_{s 1} e^{\omega t}=\frac{1}{4} u_{1}+\frac{3}{4} v_{s 1} e^{\omega t} .
\end{aligned}
$$

On the other hand, we have

$$
\begin{aligned}
& u_{2}=v_{0}+v_{1} e^{\omega t}, \quad u_{1}=-v_{f f}, \\
& v_{o}=-\frac{1}{4} v_{f f} .
\end{aligned}
$$

Hence, we get

$$
v_{1}=\frac{3}{4} v_{s 1} .
$$

The gas pressure of the pre-shock gas near the shock surface is given by 


$$
\begin{aligned}
P^{\prime} & =\frac{3}{4} \rho_{1}{ }^{\prime} u_{1}{ }^{2} \\
& =\frac{3}{4} \rho_{a}\left(u_{1}-v_{s 1} e^{\omega t}\right)^{2} \\
& \approx \frac{3}{4} \rho_{a} u_{1}^{2}-\frac{3}{2} \rho_{a} u_{1} v_{s 1} e^{\omega t} \\
& =\frac{3}{4} \rho_{a} v_{f f}^{2}+\frac{3}{2} \rho_{a} v_{f f} v_{s 1} e^{\omega t} .
\end{aligned}
$$

Since

$$
P^{\prime}=P_{0}+P_{1} e^{\omega t},
$$

we have

$$
P_{1}=\frac{3}{2} \rho_{a} v_{f f} v_{s 1} .
$$

